\title{
Idiopathic renal replacement lipomatosis: A diagnostic and therapeutic challenge
}

Gajanan Bhat ${ }^{1}$ D, Vijaykumar Barude ${ }^{1}$, Anuradha $S^{1}$, Vinayak Tembadamani', Suman Hegde ${ }^{2}$

\begin{tabular}{ll}
\hline ABSTRACT & Renal replacement lipomatosis is a rare benign renal disorder where the renal parenchyma is replaced by ma- \\
ture fatty tissue. It poses a diagnostic challenge to the clinicians due to an unusual presentation and clinical \\
features. The disease usually presents in middle age with vague pain in the affected flank and variable urological \\
symptoms. Although the condition is preceded by calculus disease or chronic inflammation, as an extremely \\
rare occurrence, it can occur without any pre-existing condition, as in our case. Increasing awareness among the \\
surgeons about the existence of such a condition will help the surgical fraternity to diagnose and manage such \\
cases properly. \\
Keywords: Benign, lipomatosis, inflammation, pyelonephritis, nephrectomy
\end{tabular}

ORCID ID of the author: G.B. 0000-0003-2067-9383

\section{Cite this paper as:}

Bhat G, Barude V, Anuradha $S$, Tembadamani V, Hedge S. Idiopathic renal replacement lipomatosis: A diagnostic and therapeutic challenge. Turk J Surg 201834 (3): 250-252.

'Division of Urology, Department of General Surgery, TSS Shripad Hegde Kadave Institute of Medical Sciences, Sirsi, India

${ }^{2}$ Department of Pathology, Shri Mahalaxmi Memorial Hospital, Sirsi, India

\section{Corresponding Author} Gajanan Bhat

e-mail: gajubhatru@gmail.com

Received: 18.09.2015

Accepted: 01.12.2015

Available Online Date: 03.01.2018

c) Copyright 2018

by Turkish Surgical Association

Available online at

www.turkjsurg.com

\section{INTRODUCTION}

Idiopathic renal replacement lipomatosis is an extremely rare benign condition of the kidney wherein the kidney parenchyma is replaced by mature adipocytes. The replacement process usually starts from the renal sinus to proceed toward the progressive involvement of the renal parenchyma and finally the perirenal space. It usually presents as a mass, posing a diagnostic challenge to the clinician. Here we present one such case in a middle-aged lady.

\section{CASE PRESENTATION}

A 45-year-old woman presented to our outpatient department with complaints of dull aching pain in the left flank of 2 years without any urinary complaints. The patient was obese with no positive findings on clinical examination duration, except for minimal left renal angle tenderness only on deep pressure. The case was presented after obtaining written informed consent from the patient.

Patients' blood and biochemical investigations were within normal limits. Ultrasound of the abdomen and pelvis could not provide any clue toward a definitive diagnosis other than showing a distorted contracted left kidney. Computed tomography (CT) scan of the abdomen revealed distorted, shrunken left kidney with the thinned-out cortex. There was loss of corticomedullary differentiation and delayed excretion of contrast. The kidney was surrounded by perinephric fat, which had pushed the abdominal organs inferiorly and medially (Figure 1). This information did not help in a definitive preoperative diagnosis. Hence, the retroperitoneum was explored through a flank incision to find a fatty mass in the left renal fossa. Due to the densely adherent mass in that region, we performed a subcapsular nephrectomy to retrieve a contracted and distorted fatty mass with very little vascularity, which could be recognized as the kidney only by the fact there were some structures of the pelvicalyceal system in the region of renal sinus and its connection to the ureter (Figure 2).

Histopathological examination revealed total replacement of the renal parenchyma with fat with very few sclerosed glomeruli with thyroidized tubules (Figure 3). The diagnosis of renal replacement lipomatosis could be made only after extensive literature study as the gross and microscopic features aptly fitted the features described in literature.

\section{DISCUSSION}

Renal replacement lipomatosis is a degenerative process that consists of progressive replacement of renal parenchyma by mature fatty tissue. It is a rare form of end stage kidney condition resulting from chronic inflammation. The condition was unheard of till Kurtzmann described it in detail during his study of 33 cases in 1931 (1). Even now, the condition is rarely seen in clinical practice wherein it presents a diagnostic and management challenge.

The disease usually presents in the fourth and fifth decades of life, although there are cases reported in the pediatric age group too. The usual presentation is in the form of a vague pain in the involved 


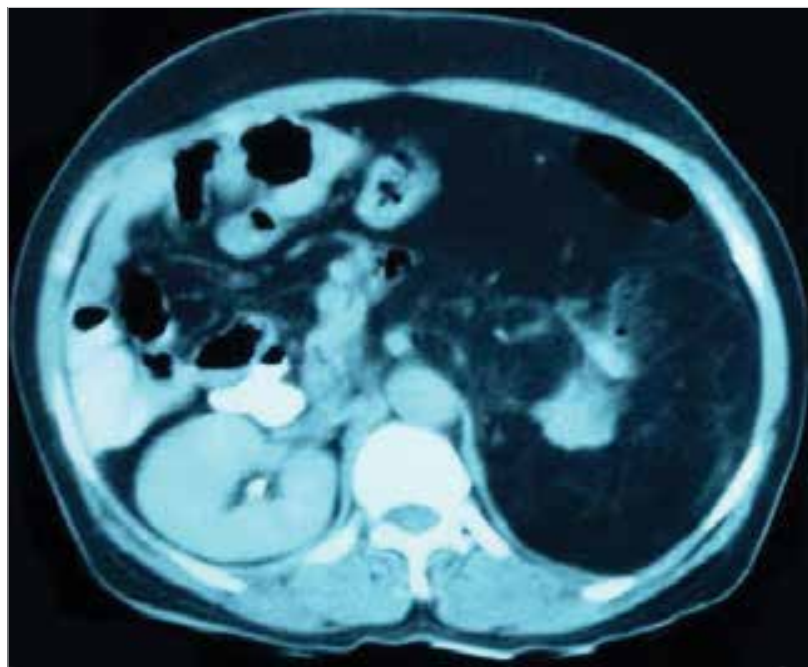

Figure 1. Computed tomography scan abdomen picture showing distorted left kidney surrounded by fat pushing abdominal content inferomedially

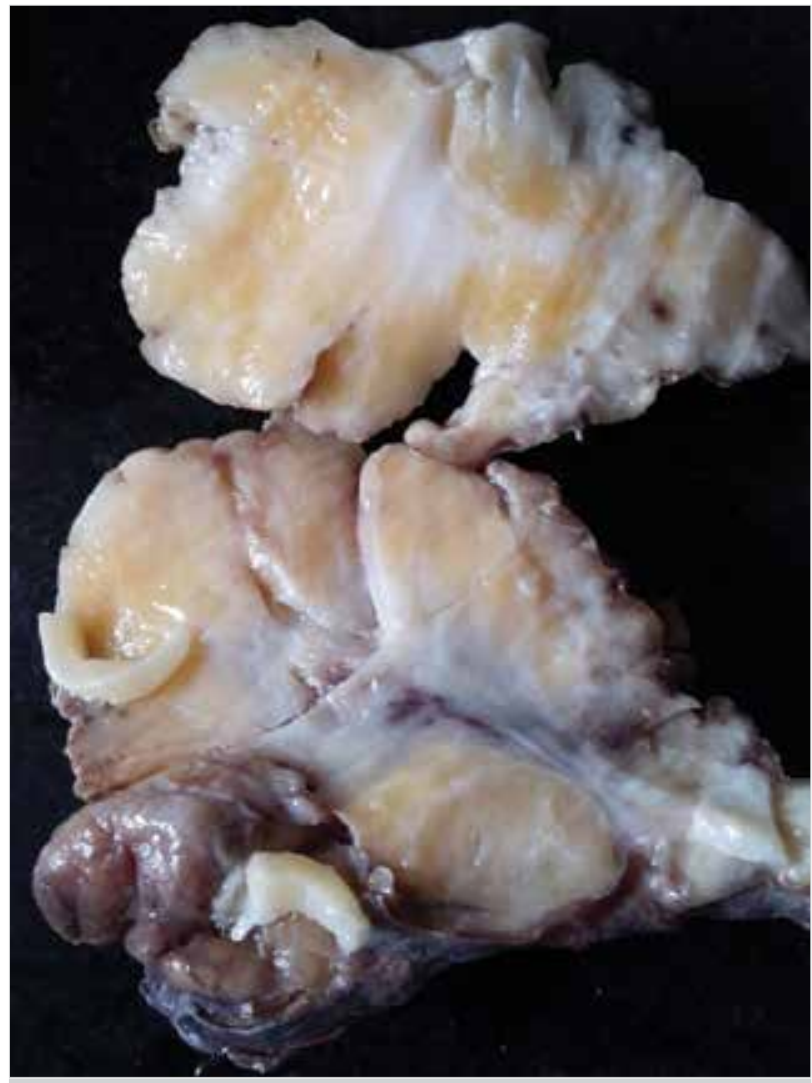

Figure 2. Cut surface of the specimen

flank with variable urinary symptoms. Although the condition is preceded by either a calculus disease or chronic pyelonephritis, there are very rare instances where no such preceding condition exists, such as ours $(1,2)$. It is important to promptly diagnose this condition as there are reports of coexisting malignancy too (3). Clinical examination hardly yields any positive finding.

In most of the cases reported, preoperative diagnosis could not be made. This is due to the paucity of classical features either on clinical examination or on investigation. Routine ultrasound of the abdomen can detect distorted renal paren-

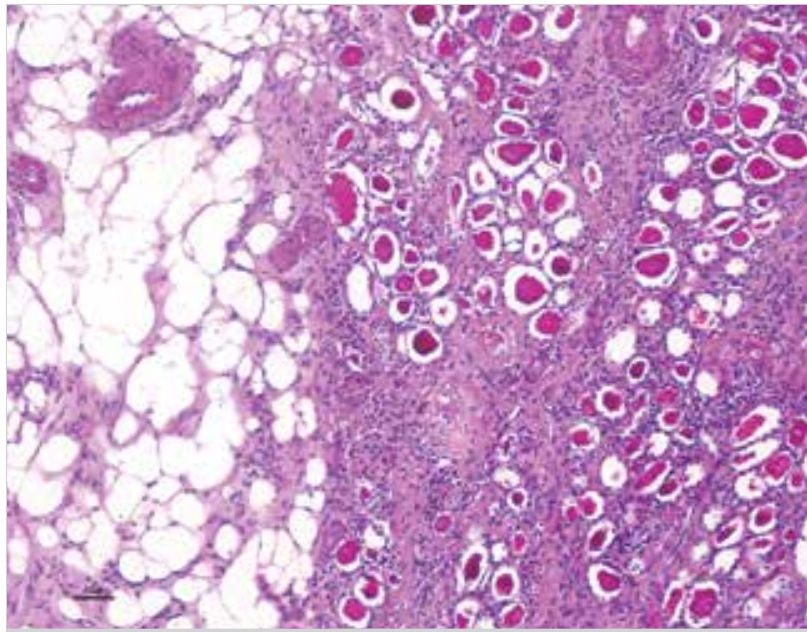

Figure 3. Photomicrograph of the histopathological examination of the specimen showing mature adipocytes, sclerosed glomeruli, thyroidized tubules, and inflammatory infiltrates (H\&E stain, size: $40 \mu \mathrm{m}$ )

chyma and presence of calculus. CT can further characterize the lesion as a fatty infiltration of the poorly functioning renal moiety. Magnetic resonance imaging can add to anatomic details (4). However, none of these can definitely point to the diagnosis of renal replacement lipomatosis.

As of today, histopathology is the only accurate diagnostic tool. The kidney is usually shrunken in size as in our case, although it may be enlarged as in cases associated with calculus disease and there is decreased vascularity of the renal parenchyma with replacement of parenchyma by mature adipocytes. There is infiltration by the inflammatory cells to certain extent. Xanthogranulomatous pyelonephritis is the closest mimicker of this condition, especially in case of calculus. The absence of xanthoma cells confirms diagnosis in favor of renal replacement lipomatosis $(2,4)$.

Differential diagnoses other than xanthogranulomatous pyelonephritis are malakoplakia and fat-containing tumors of the kidney and perirenal structures. Malakoplakia is characterized by Michaelis-Gutmann bodies, which are foamy histiocytes with basophilic inclusions. The diagnosis of malakoplakia is also made by histopathological examination in almost all cases, and there are suggestions that malakoplakia is also a stage in the formation of xanthogranulomatous pyelonephritis $(5,6)$.

The fat-containing tumors of the kidney such as angiomyolipoma and liposarcoma usually arise from the sinus fat and not from the renal parenchyma, hence cause mass effect rather than renal parenchymal lesions. The mass effect can cause vague pain and sometimes obstructive features when the mass causes obstruction at the level of the renal pelvis. They are usually identifiable by their specific imaging features unlike renal replacement lipomatosis, which has no specific features on imaging that can confirm diagnosis (6).

Preoperative diagnosis is important as it is challenging to plan the treatment for this condition. As such renal replacement lipomatosis itself is not of much clinical significance as it denotes a degenerative process. The rarity of its occurrence and 
paucity of literature in contemporary medical textbooks about the condition adds to the difficulty of the clinician to diagnose the condition preoperatively. Although it is easy to diagnose a fatty mass on $\mathrm{CT}$, to characterize this entity preoperatively as renal replacement lipomatosis is a clinical challenge. In almost all reported cases, the diagnosis was confirmed on histopathology only (1-6). Hence, the best treatment in such cases is excision of fatty mass intotal. However, this may not be possible due to severe adhesions, as in our case.

\section{CONCLUSION}

The rarity, nonspecific clinical features, and absence of classical investigative findings in case of renal replacement lipomatosis pose a diagnostic and therapeutic challenge to clinicians. With reporting of cases of all types and variants of this clinical condition, awareness about renal replacement lipomatosis can increase among clinicians, radiologists, and pathologists, which will help in accurate preoperative diagnosis and treatment planning.

Informed Consent: Written informed consent was obtained from patient who participated in this study.

Peer-review: Externally peer-reviewed.

Author Contributions: Concept - G.B., A.S., S.H.; Design - G.B., V.B.; Supervision - G.B., V.T.; Resource - G.B., S.H.; Materials - G.B., V.B., S.H.; Data
Collection and/or Processing - G.B., A.S.; Analysis and/or Interpretation - A.S.; Literature Search - G.B., S.H.; Writing Manuscript - G.B.; Critical Reviews - A.S., V.T.

Conflict of Interest: The authors have no conflicts of interest to declare.

Financial Disclosure: The authors declared that this study has received no financial support.

\section{REFERENCES}

1. Peacock AH, Balle A. Renal lipomatosis. Ann Surg 1936; 103: 395401. [CrossRef]

2. Shah VB, Rupani AB, Deokar MS, Pathak HR. Idiopathic renal replacement lipomatosis: A case report and review of literature. Indian J Pathol Microbiol 2009; 52: 552-553. [CrossRef]

3. Arora P, Rao S, Khurana N, Ramteke VK. Renal replacement lipomatosis with coexistent papillary renal cell carcinoma, renal tubulopapillary adenomatosis and xanthogranulomatous pyelonephritis: an extremely rare association and possible pathogenetic correlation. Urol J 2013; 10: 906-908.

4. Prasad KR, Chandra HS, Vijay Kumar KR. Renal replacement lipomatosis. Indian J Urol 2012; 28: 105-106. [CrossRef]

5. Yigter M, Ilgici D, Celik M, Arda IS, Hiscsonmez A. Renal Parenchymal malakoplakia: a different stage of xanthogranulomatous pyelonephritis? J Pediatric Surg 2007; 42: E35-38. [CrossRef]

6. Ginat DT, Bhatt S, Dogra VS. Replacement lipomatosis of the kidney. J Ultrasound Med 2008; 27: 1393-1395.[CrossRef] 